Cahiers $d u$ MONDE RUSSE

\section{Cahiers du monde russe}

Russie - Empire russe - Union soviétique et États indépendants

$51 / 4 \mid 2010$

Sciences humaines et sociales en Russie à l'Âge d'argent

\title{
Elizabeth White, The Socialist Alternative to Bolshevik Russia
}

\section{Thomas Chopard}

\section{(2) OpenEdition}

1 Journals

Édition électronique

URL : https://journals.openedition.org/monderusse/7379

DOI : $10.4000 /$ monderusse. 7379

ISSN : $1777-5388$

Éditeur

Éditions de l'EHESS

\section{Édition imprimée}

Date de publication : 25 novembre 2010

Pagination : 696-700

ISBN : 978-2-7132-2316-7

ISSN : $1252-6576$

Référence électronique

Thomas Chopard, «Elizabeth White, The Socialist Alternative to Bolshevik Russia », Cahiers du monde russe [En ligne], 51/4 | 2010, mis en ligne le 09 décembre 2011, consulté le 03 septembre 2022. URL : http://journals.openedition.org/monderusse/7379; DOI : https://doi.org/10.4000/monderusse.7379

Ce document a été généré automatiquement le 3 septembre 2022

Tous droits réservés 


\title{
Elizabeth White, The Socialist Alternative to Bolshevik Russia
}

\author{
Thomas Chopard
}

\section{RÉFÉRENCE}

Elizabeth WHITE, The Socialist Alternative to Bolshevik Russia. The Socialist

Revolutionary Party, 1921-1939. Londres : Routledge, 2011, 180 p.

Dans ce premier livre concis, Elizabeth White expose une partie des éléments traités dans sa thèse qui porte plus généralement sur l'émigration russe en Tchécoslovaquie. Le titre est en effet trompeur : l'ouvrage ne concerne pas le parti socialiste-révolutionnaire (SR) en Russie, ni même globalement ce parti en émigration, mais un groupe restreint d'une quarantaine de militants exilés en Tchécoslovaquie et particulièrement actifs au sein de diverses publications (périodiques, brochures, livres, etc.).

2 Si l'ouvrage se place au croisement de l'histoire de l'émigration russe et de celle des partis socialistes antibolcheviks - toutes deux connaissant un certain regain d'intérêt -, il prend toutefois le parti d'analyser principalement les textes étudiant la situation soviétique et théorisant les prises de position politiques issus de la branche praguoise du parti SR. Et cette analyse apparaît essentiellement comme celle d'un groupe constitué dont les différents membres sont peu ou pas mis en relief, à l'exception notable de Viktor Černov, ministre de Kerenskij et président de l'éphémère Constituante de 1918.

De ce fait, Elizabeth White organise son propos selon un fil chronologique correspondant aux grandes dates de l'histoire soviétique. Le premier chapitre porte ainsi sur la période d'avant l'émigration, c'est-à-dire sur l'héritage narodnik et la révolution; le deuxième traite de l'arrivée en Tchécoslovaquie et de l'opposition avec l'autre pôle majeur du parti SR : Paris; les deux suivants ont trait à la mise en place de la NEP et au mode de gouvernement bolchevik; le quatrième chapitre envisage la question des nationalités sous l'angle de la rupture entre Černov et les SR de Prague sur ce point. Les sections VI et VII abordent l'arrivée de Stalin au pouvoir, qui suit de peu l'élimination des derniers 
SR encore organisés en URSS, puis la collectivisation. Le huitième et dernier chapitre est à part, puisqu'il traite des conditions de vie et du destin, au cours des années 1930 et 1940, des SR de Prague qui se retrouvent rapidement isolés en exil, dépourvus de contact avec l'URSS et de financement ; la fin traite largement des réactions diverses de ces SR face au fascisme et à l'invasion de l'Union soviétique ; l'après-guerre est très brièvement évoqué. Le premier chapitre offre une synthèse efficace de l'histoire du parti SR en Russie, avec son héritage narodnik et un programme politique essentiellement tourné vers les campagnes de l'Empire russe et un postulat essentiel - la commune. La révolution et la guerre civile - pourtant à l'origine de l'exil envisagé - ne sont que brièvement évoquées. Tandis que l'historiographie sur le sujet est plus que conséquente, la révolution n'est analysée qu'au prisme de l'ouvrage que Viktor Černov - alors ministre de l'Agriculture lui consacre, et du programme élaboré par le parti en 1917, c'est-à-dire uniquement au niveau des plus hauts dirigeants politiques. Cette approche, reprise au chapitre III, fait conclure à Elizabeth White que l'une « des raisons principales de l'échec du parti SR à consolider le pouvoir en 1917 » résidait dans son impéritie à établir un programme cohérent pour la société russe ; les mouvements sociaux, la guerre, n'étant quant à eux que des « vicissitudes de l'année elle-même » (p. 45).

5 Si la période révolutionnaire est ainsi décrite, c'est toutefois sans opérer de lien avec la période immédiate de l'exil qui commence en 1919. Elizabeth White souligne l'attitude accueillante de la nouvelle Tchécoslovaquie à ces exilés révolutionnaires antibolcheviks. Mais la participation notable des SR au gouvernement antibolchevik de Sibérie, soutenu notamment par la Légion tchécoslovaque constituée lors de la Grande Guerre, n'est analysée que comme un moyen de sortir de Russie et jamais comme une expérience structurante. De même, on ne sait pas si cette sortie est organisée par le gouvernement de Prague ou si les SR profitent de ce désengagement militaire massif. Ce deuxième chapitre est néanmoins surtout consacré à la mise en place de l'immigration à Prague. L'auteur esquisse de nombreuses notices biographiques de ces exilés, mais elles ne sont que peu exploitées, sinon pour présenter un tableau impressionniste de quelques parcours et positions politiques de SR, qui confine ainsi à la galerie de portraits figés. Les moyens de subsistance de ces émigrés en Tchécoslovaquie sont également énumérés (en particulier leur activité au sein du Zemgor), mais l'intégration de ces étrangers dans une société dont ils ne parlent pas la langue n'est jamais envisagée. Une même présentation rapide est opérée pour les journaux fondés par les SR de Prague, au sein desquels ces derniers sont particulièrement actifs pendant les années 1920.

Les chapitres III et IV arrivent au cœur de la démarche d'Elizabeth White en abordant l'analyse par les SR de la première métamorphose radicale de la société soviétique, la NEP, et de la nature de l'État soviétique lui-même. Il s'agit essentiellement pour les SR de Prague de démontrer la persistance du modèle de la société paysanne au sein de l'URSS, la NEP étant ainsi envisagée comme un moment de soulagement pour les campagnes. La description des campagnes que l'auteur propose à travers les analyses des SR est toutefois problématique : les paysans seraient sortis indemnes de la révolution et de la guerre civile (p.62) et constitueraient ainsi par inertie un foyer de résistance aux aspirations soviétiques, ce dont témoigneraient les violences spontanées. Si Elizabeth White insiste particulièrement sur ce phénomène d'embrasement des campagnes et de pacification forcenée, ce développement aurait mérité d'être mis en contradiction avec la position SR qui voit les campagnes comme renforcées par ce temps de troubles. À 
moins de considérer la guerre et ses millions de citadins réfugiés dans les campagnes comme un vecteur de stabilité.

Quant à la nature de l'État soviétique, elle est envisagée par les SR sous l'angle de la "modernisation", c'est-à-dire de la capacité de l'État à favoriser le développement économique du pays (le terme n'est toutefois jamais totalement explicité). La NEP est ainsi vue comme un moment de ralentissement de la modernisation bolchevique après les années de communisme de guerre, un moment notamment favorable à la paysannerie. Cette dernière dimension incite l'auteur à conclure à une porosité des idées bolcheviques aux conceptions SR des campagnes, mais une porosité seulement tactique. Cet argument, qui pourrait permettre d'envisager la NEP comme la continuation d'un rapport de force politique contradictoire après la révolution, se limite toutefois à la seule évocation de Lenin et Buharin. Ainsi, la société soviétique ne serait pas traversée de conceptions contradictoires sur son avenir et sur son développement, elle aurait seulement à sa tête des dirigeants opportunistes qui lisent la presse socialiste-révolutionnaire.

Létude de la nature de l'État soviétique se poursuit au chapitre IV, centré sur le mode de gouvernement bolchevik et sur le parti, ainsi que sur le potentiel de résistance des différentes classes sociales auxquelles les SR pourraient apporter leur concours. Le parti au pouvoir est dépeint par les SR sous les traits d'un parti militarisé, intolérant pour ne pas dire fanatisé et répressif. Malgré l'évocation des "sovburžui", on regrettera que l'auteur n'ait pas mis en regard de ces appréciations l'évolution rapide par bureaucratisation des instances communistes. L'analyse SR s'en tient donc aux grandes figures du parti, expliquant les variations de la politique soviétique au début des années 1920 par une psychologie simpliste (p.71-72). Si les SR de Prague ont une appréciation particulièrement négative du parti communiste, ils se montrent tout aussi déçus des résistances et des réactions des autres catégories de la population qu'Elizabeth White passe en revue : paysannerie, intelligentsia, prolétariat. Partout les SR semblent isolés. La seule solution envisagée pour y remédier est, conformément à l'héritage narodnik, d'infiltrer les instances paysannes locales. L'ouvrage montre de manière récurrente l'isolement progressif des SR en exil et le lecteur décèle, particulièrement dans ce chapitre, un décalage croissant entre leurs analyses et les revendications ou résistances réelles des citoyens soviétiques.

Les chapitres VI et VII marquent une double rupture : d'une part, ils correspondent aux analyses couvrant l'émergence du stalinisme, d'autre part ces années s'accompagnent du démantèlement des vestiges du parti SR en URSS à partir de 1925. Ces arrestations isolent d'autant plus les émigrés. Elizabeth White ne déploie toutefois pas suffisamment les canaux d'information entre l'Union soviétique et la Tchécoslovaquie pour permettre au lecteur de savoir précisément comment cet événement soviétique entrave la production intellectuelle des SR de Prague, ni comment il instille un climat de suspicion au sein du groupe.

L'analyse du stalinisme est envisagée dans l'ouvrage sous deux angles : l'arrivée de Stalin au pouvoir et la collectivisation. Le premier est largement mésestimé : les SR de Prague $\mathrm{y}$ voient une très grave division et certains, dont Černov, en viennent à prophétiser de façon optimiste la fin de l'URSS en tant que régime répressif. Le même constat sera repris par les SR lors des nombreux procès des années 1930 dans lesquels ils sont souvent cités. Les exilés prennent au fond les accusations au sérieux et considèrent les procès comme le signe d'une lutte accrue contre le régime soviétique, menée par d'anciens SR, les " spécialistes bourgeois », l'Armée rouge, etc. 
11 Malgré le voyage clandestin d'un SR en URSS en 1929, longuement évoqué par Elizabeth White, les appréciations des conséquences économiques du stalinisme sont inattendues: les effets du plan quinquennal sur l'économie ne sont considérés que comme une brève crise économique. C'est surtout sur la collectivisation que l'auteur s'attarde: pour les émigrés, le problème fondamental est de savoir si elle est volontaire ou non; la confusion règne en effet quant au terme lui-même, car, dans la tradition narodnik, la nationalisation des terres est perçue comme un «but élevé » (p. 115). De ce fait, peu de SR soutiendront les résistances qui accompagnent la collectivisation. D'une façon générale, l'ensemble du parti SR, lors de son congrès en 1931, a du mal à appréhender les transformations radicales apportées par la collectivisation au monde paysan: l'idéal de la commune paysanne est maintenu, et la collectivisation aussi bien que l'industrialisation massive due au plan ne sont que faiblement intégrées dans le programme issu de ce congrès.

12 Le lecteur impute spontanément cette interprétation du stalinisme à un isolement croissant, à un manque d'informations directes sur la situation soviétique et surtout à un déséquilibre au sein du parti SR fonctionnant désormais seulement en exil. L'auteur ne l'explique toutefois que dans le cas de Viktor Černov, et par la seule dimension psychologique : l'exil et la maladie feraient qu'il «interprète les événements avec une approche trop théorique » (p. 110).

13 Les chapitres V et VIII traitent moins de la situation soviétique que des conflits internes à l'émigration. Le premier explore la question de la Ligue socialiste du Nouvel Est et le problème des nationalités, tandis que le dernier traite du destin des SR de Prague dans le contexte européen des années 1930-1940.

14 La question des nationalités est l'occasion d'un premier effritement du groupe des SR exilés en Tchécoslovaquie. Certains, souvent d'origine ukrainienne ou géorgienne, mettent en avant le caractère colonial de la présence russe dans les confins de l'ancien Empire, tandis que de nombreux SR conçoivent toujours la Russie comme un pays multiethnique. Elizabeth White montre très clairement le poids de l'héritage tsariste dans la conception des nationalités propre aux SR, un poids exacerbé par les indépendances - parfois avortées - de la révolution, et dont les tensions sont reprises dans le cadre de cette Ligue socialiste du Nouvel Est favorable à un programme antiindépendantiste qui voit se rapprocher certains SR de Prague de ceux de Paris, également anti-indépendantistes.

Le destin des SR se complexifie largement après 1932, sans que les conditions matérielles soient particulièrement analysées par Elizabeth White. Pourtant, si ces années cruciales pour l'Europe n'occupent qu'un chapitre, c'est parce que les SR sont rapidement coupés de toute source de financement. Face à la montée du fascisme, la Tchécoslovaquie, autrefois accueillante envers ces SR émigrés, se range du côté de l'URSS en 1935 et tait ses faveurs socialistes-révolutionnaires. Zemgor, journaux, etc., disparaissent rapidement. Qui plus est, les menaces de guerre amènent certains SR à défendre malgré tout la Russie en danger et à atténuer par là même leurs critiques, voire à chanter les louanges de l'Armée rouge, seul rempart face aux agressions extérieures. L'auteur passe hélas rapidement sur ces trajectoires multiples au travers de l'Europe en proie au fascisme, qui amènent par exemple d'anciens militants antibolcheviks à combattre dans les rangs des partisans yougoslaves durant la Seconde Guerre mondiale ; ou, au contraire, à émigrer aux États-Unis. De même, l'emprisonnement des rares émigrés restés à Prague 
après la guerre aurait peut-être mérité plus de détails. Cette recomposition radicale du groupe d'émigrés initial dans les années 1930 nous paraît mériter plus qu'un épilogue.

Ce dernier chapitre nous semble révélateur du problème essentiel que pose l'ouvrage d'Elizabeth White, à savoir le parti pris intellectualiste de l'auteur, qui écarte toute la dimension matérielle de l'émigration, tant comme sujet d'étude que comme facteur explicatif. De manière plus problématique encore, les idées étudiées ne le sont pas comme un objet historique, porté par des acteurs et inséré dans des discussions voire des conflits : elles sont mesurées à la seule aune de la vérité. Et ce, comme il est annoncé dès l'introduction, parce que «l'étude du parti socialiste-révolutionnaire en émigration est d'une importance particulière pour comprendre l'histoire de la Russie au $\mathrm{XX}^{\mathrm{e}}$ siècle » (p. 2). Les SR de Prague ne sont pas un objet de recherche, ils sont un outil pour l'historien, car de fait « les SR soulèvent les mêmes questions [...] que les chercheurs (scholars) aujourd'hui » (p. 4). Or un énoncé, même s'il tend dans tous les cas à décrire une situation, nous semble relever de deux régimes de discours radicalement différents s'il est produit dans le cadre d'un journal d'opposants politiques des années 1920 ou dans un livre d'histoire. Qualifier un discours de politique et en montrer les déterminations ne nous semble pas pour autant le condamner au mensonge.

L'auteur compare sa méthode à celle employée par «Shanin, Lewin et Danilov [qui] ont montré que les bolcheviks avaient une compréhension inadéquate des dynamiques de la société paysanne », tandis que les SR avaient raison (p. 142). La différence entre les deux approches est toutefois importante: ces auteurs semblent avoir étudié les méthodes bolcheviques d'appréhension du monde paysan non pour elles-mêmes, mais en vue de montrer quelles représentations avaient guidé les décisions et l'action politiques. Afin de " comprendre l'histoire de la Russie au $\mathrm{xx}^{\mathrm{e}}$ siècle », il semble plus pertinent de saisir pourquoi les instances dirigeantes du pays avaient tort et comment elles fonctionnaient, plutôt que d'analyser comment quarante émigrés en Tchécoslovaquie avaient raison. Car les SR de Prague ne sont plus dans l'action politique, mais dans l'inaction subie, dont découle leur production analytique. Si alternative SR il y a eu, ce fut probablement pendant la révolution, pas après.

Ce parti pris intellectualiste sous-tend l'ouvrage tout entier qui oscille parfois entre l'étude et la pure et simple paraphrase des sources sans aucun recul critique. Cette posture fait presque regretter que l'auteur n'ait pas préféré produire un recueil de documents substantiel comme Vladimir Brovkin a pu le faire avec les mencheviks ${ }^{1}$.

Point de détail, enfin: Elizabeth White légitime son étude par le fait que cette presse SR de Prague était « la seule presse russe libre de l'entre-deux-guerres». On soulignera que le fait d'être presque entièrement financé par un gouvernement étranger - en l'occurrence tchécoslovaque - est rarement synonyme d'indépendance.

\section{NOTES}

1. Vladimir Brovkin, Dear Comrades: Mensheviks reports on the Bolshevik revolution and the civil war, Stanford : Hoover Institution Press, 1991. 